\title{
VARIA
}

\section{NOTICIAS DOCUMENTALES SOBRE EL COLEGIO DE SAN BARTOLOMÉ DE SALAMANCA}

La construcción del colegio de San Bartolomé o de Anaya, el más antiguo e importante de los colegios universitarios españoles al haber sido fundado en Salamanca hacia 1410 por don Diego de Anaya a imitación del de San Clemente de Bolonia, no ha sido estudiada con la atención que merece. Ello se ha debido probablemente a la extraña carencia de documentación. Con seguridad la hubo pero se debió perder en el trasiego de papeles que fueron incautados y enviados a Madrid durante el proceso de reformas que ordenó efectuar en 1771 Carlos III ${ }^{~}$. Aunque algunos de esos legajos fueron devueltos a la Universidad de Salamanca no hace mucho tiempo, no se han encontrado en su rico archivo los pertinentes a la construcción, si bien pudiera suceder que no hubieran sido hasta ahora catalogados en su integridad.

Las escasas noticias que hay sobre este asunto se deben a don José Roxas y Contreras, marqués de Alventos, quien compuso entre 1666 y 1670 tres volúmenes sobre la historia del colegio, pero cuyo interés se polarizó en la defensa de sus constituciones, estatutos, derechos y privilegios históricos, amenazados por el proceso de reformas, y no precisamente sobre el elogio del nuevo edificio en construcción, paralizado precisamente a causa de los dispendios que generaba ${ }^{2}$. El intento de estas sucintas notas es reunir las pocas y dispersas referencias documentales que hay sobre esta materia en los archivos de Salamanca y en el General de Simancas.

Según el citado marqués de Alventos el edificio original del colegio fue más bien pobre, hecho de mampostería y tapiales de ladrillo, sin pretensión artística alguna y muy lejos de la magnificencia del que se construyó a partir de 1760 y que es el que hoy subsiste. Efectivamente una viñeta que ilustra la mencionada historia muestra el humilde aspecto que ofrecía el viejo edificio costeado por don Diego de Anaya, y eso que durante el siglo xvi había sido ampliado con una regular escalera y fortificado con estribos gracias a la generosidad del cardenal Silíceo, arzobispo de Toledo y antiguo colegial de San Bartolomé, quien entregó para ello 2.000 ducados ${ }^{3}$. Con todo un siglo después, en 1670 , el edificio presentaba tremendas grietas y desplomes en el ángulo de encuentro de sus lados oriental y septentrional y menores en otros lien-

' Sala Balust, Luis, "Catálogo de fuentes para la historia de los antiguos colegios seculares de Salamanca», Hispania Sacra, 7, 1954, pp. 1-124; Id., Colegios de Salamanca, 1623-1770, Universidad de Valladolid, Valladolid 1956; Id., Visitas y reformas de los Colegios Mayores de Salamanca en reinado de Carlos III, Universidad de Valladolid, Valladolid 1958.

${ }^{2}$ Roxas y Contreras, José, Historia del Colegio Viejo de San Bartolomé, Mayor de la célebre Universidad de Salamanca..., 3 vols., A. Ortega, Madrid 1766-1770.

${ }^{3}$ Ibid., vol. III, pp. 307-310. 
zos de pared, como se dedujo de un reconocimiento hecho a instancias del rector, don Francisco Esquivel, por el maestro mayor de la vecina catedral Juan de Setién Güemes. Su informe fue ratificado por el de otros dos arquitectos convocados por el obispo, don Gabriel de Esparza, a saber el jesuita Pedro Mato, que construía la iglesia de la Clerecía, y el conocido agustino recoleto fray Lorenzo de San Nicolás, que se encontraba en la ciudad para dictaminar sobre el desplome de la cúpula de la iglesia de las Agustinas. La solución fue reforzar aún más los viejos muros entibándolos con nuevos contrafuertes de piedra, operación que efectuó Juan de Setién, cobrando 24.000 reales ${ }^{4}$.

No debió servir de mucho este segundo reforzamiento del ruinoso edificio por cuanto otro siglo después hubo la precisión de derribarlos, cuarteado como había quedado por el terremoto de Lisboa de 1755, para dar paso a uno totalmente nuevo. Pero ya antes, a comienzos del XvIII, se había comenzado a ampliar su núcleo principal por oriente con la hospedería y por occidente con la iglesia de San Sebastián. La hospedería se levantó en una cota mucho más baja a causa de la pendiente que bajaba bruscamente hacia el arroyo de Santo Domingo. Debió comenzarse hacia 1702, costeada por el antiguo colegial don Juan de Isla, arzobispo de Burgos. Aunque hay constancia de la intervención en la obra de Pantaleón del Pontón Setién, sobrino de Juan Setién, a su muerte acaecida en 1713 le sucedió Joaquín de Churriguera, a quien se debe particularmente su hermoso patio interior. Por otro lado se sabe por documento exhumado por el profesor Jaime Pinilla que en 1716 se estaban poniendo las armaduras de madera de los tejados y se aprestaba la teja necesaria para cubrirlos ${ }^{5}$. Es la hospedería un sencillo edificio cuadrangular, compuesto de un sótano y dos plantas, y realizado íntegramente con sillería arenisca de Villamayor a fin de garantizar su perdurabilidad. Apenas moldurado en el exterior, que ofrece un aspecto meramente utilitario, su mayor atractivo se centra en el patio interior de dos pisos, inspirado en el renacentista del colegio del Arzobispo en una suerte de afán retrospectivo, que ya G. $\mathrm{Ku}$ bler señaló como una de las características de la obra de los Churriguera.

Del otro lado del viejo colegio se levantó la iglesia pública, que vino a englobar la fenecida parroquia de San Sebastián, ya que aquel sólo tenía una capilla privada consagrada a San Juan de Sahún, uno de sus colegiales fundadores. Las actas de la vecina catedral daban cuenta el 9 de noviembre de 1731 de que el rector y colegiales de San Bartolomé estaban fabricando iglesia con el ánimo de unir a ella la parroquia de San Sebastián, vinculada desde antiguo al colegio en virtud de Bula Pontificia. Para ello solicitaban la aquiescencia del obispo, don José Sancho Granados y del cabildo ${ }^{6}$. Este documento fija la fecha «ante quem» de la construcción de la iglesia. Al año siguiente, el 17 de octubre, el cabildo vende al colegio unas canteras sitas en Villamayor «para la continuación de su obra de la iglesia», así como permuta las casas y oficinas del deán necesarias para «la capilla del colegio viejo que tiene levantadas las paredes, pues hacen medianería con dichas casas» ${ }^{7}$. Finalmente el colegio hizo saber el 29 de abril de 1739 que, terminada la obra de la iglesia, estaba a punto de trasladar a ella el Santísimo Sacramento y de demoler la vieja capilla de San Juan de Sahún, invitando al cabildo a su inauguración ${ }^{8}$. Por consiguiente la construcción de la iglesia se desarrolló entre 1730 y 1739, corriendo a cargo de Alberto de Churriguera, quien también dirigía entonces las obras de la catedral, imprimiéndole tanto exterior como interiormente su huella inconfundible. En recuerdo de San

\footnotetext{
${ }^{4}$ Archivo Histórico Provincial de Salamanca, protocolo de Pedro Mendoza Carrillo 3.018, fols. 447-448v.

${ }^{5}$ Rodríguez G. de Ceballos, Alfonso, Estudios del Barroco Salmantino II: el Colegio de la Orden Militar de Calatrava de la Universidad de Salamanca, Centro de Estudios Salmantinos, Salamanca 1972, pp. 12-15. Joaquín de Churriguera heredó de Pantelón de Pontón Setién tanto la maestría de la obra del colegio de Calatrava como la de la hospedería del colegio de Anaya.

${ }^{6}$ Archivo de la catedral de Salamanca, Registro de Actas capitulares no 52, 1730-1740, fol. 31, r-v.

${ }^{7}$ Ibid., fol. $252 \mathrm{r}$

${ }^{8}$ Ibid., fol. $733 \mathrm{v}$
}

AEA, LXXVI, 2003, 302, pp. 187 a 205 

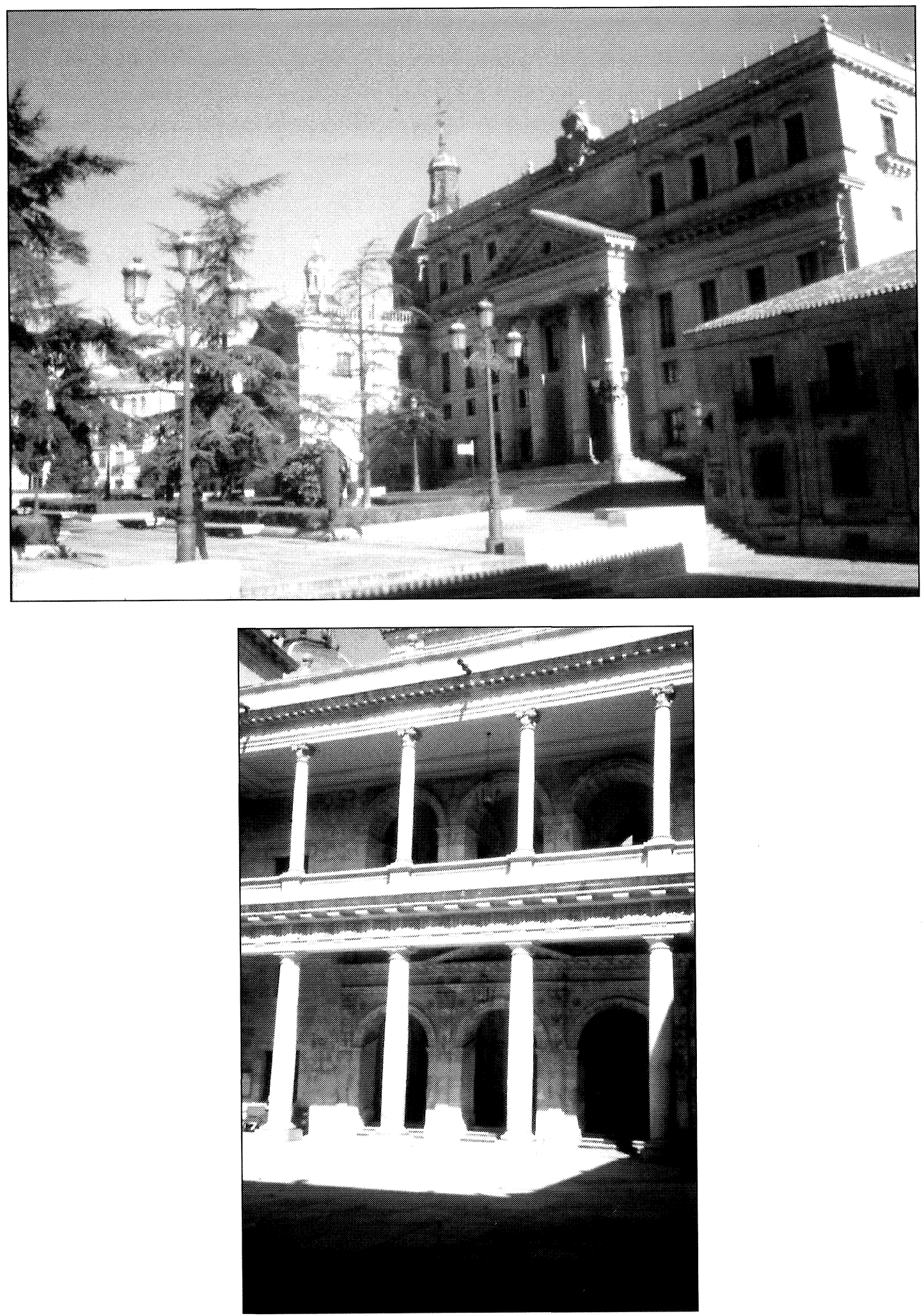

Fig. 1. Fachada del Colegio nuevo de San Bartolomé de Salamanca.

Fig. 2. Patio del Colegio de San Bartolomé. Al fondo, entrada a la escalera.

AEA, LXXVI, 2003, 302, pp. 187 a 205 
Juan de Sahún, cuya primitiva capilla se había tenido que demoler, se dedicó una de las portadas de la nueva iglesia a este santo, a saber la lateral que se abre en el costado de la epístola, colocando sobre ella su estatua, pues la principal está advocada a San Sebastián, titular de la parroquia.

El primer retablo de esta iglesia, realizado en madera, se componía de una custodia o expositor en el primer cuerpo, flanqueada por episodios de la vida de San Sebastián hechos de pintura por Pedro de Tordesillas y Pedro de Aguilar, mientras en el segundo había un Crucifijo de talla, siendo los soportes de orden corintio a causa de la vistosidad de su capitel. Ignoramos quien fuese el ensamblador de este retablo, pero no quien lo doró y policromó por contrato de 16 de junio de 1756, a saber Francisco Báez ${ }^{9}$. Parece que fue desplazado por otro de mármoles y jaspes, del que realizó únicamente la mesa del altar, el sagrario, las gradas y el expositor Simón Gavilán Tomé. El que actualmente ostenta la iglesia se hizo en 1903 por iniciativa del obispo don Tomás Cámara y Castro, aprovechando un magnífico lienzo del martirio de San Sebastián pintado en 1740 por Sebastiano Conca, lienzo que se hallaba depositado en el Museo Provincial de Bellas Artes ${ }^{10}$.

El nuevo edificio del colegio, a tenor de la escasa documentación disponible, debió ser trazado en 1760 por José de Hermosilla y Sandoval recomendado para este fin por su hermano Ignacio, secretario de la Real Academia de Bellas Artes de San Fernando y antiguo colegial de San Bartolomé. Hermosilla había abandonado la dirección de la enseñanza de la arquitectura en la Academia en 1765, reincorporándose al cuerpo de ingenieros militares de donde procedía, pero aquella le siguió encomendando importantes encargos ${ }^{11}$. No sabemos si el ilustre ingeniero estuvo en Salamanca pues A. Ponz asegura que únicamente envió los dibujos ${ }^{12}$, cuya materialización correspondió a Juan de Sagarvinaga, maestro de obras de la catedral salmantina desde enero de 1755 , persona muy respetuosa en la ejecución de trazas ajenas cuanto carente de inventiva en las propias. La obra no parece que saliese de cimientos antes de 1762 . La construcción quedó interrumpida en abril de 1771, nueve años después de empezada, de orden de los comisionados reales nombrados por Carlos III en 25 de febrero para ejecutar las reformas de los colegios universitarios. Se inició entonces una nutrida correspondencia entre don Manuel de Roda, ministro de Gracia y Justicia, y el obispo de Salamanca don Felipe Beltrán, designado concretamente visitador de los colegios mayores salmantinos ${ }^{13}$.

Por lo pronto el entonces rector del colegio de San Bartolomé, don Benito de la Mancha Linares y otros dos colegiales representaron al prelado el 28 de mayo amarga queja por la interrupción de la obra y especialmente de su grandiosa escalera, punto por el que habían sido denunciados por el cabildo catedralicio, puesto que para realizar su enorme caja se habían tenido que expropiar forzosamente algunas casas de su propiedad. Según el rector y los colegiales la construcción de un nuevo edificio fue de obligada necesidad, pues según declaración de los arquitectos consultados al efecto el antiguo no podía sostenerse más en pie a causa de su mucha antigüedad y del daño que padeció con ocasión del terremoto de 1755. En cuanto a la dispendiosidad de la obra, de que se les acusaba, «no puede dudarse — decían — de la mucha utilidad que resulta de ella tanto a la comodidad de los individuos como al público en la cons-

\footnotetext{
${ }^{9}$ Archivo Histórico Provincial de Salamanca, protocolo de Pedro Mendoza Carrillo 3.006, fol 632-133v.

${ }^{10}$ Rodríguez G. de Ceballos, Alfonso y Nieto González, José Ramón, «Aportaciones a Simón Gavilán Tomé», Archivo Español de Arte, 213, 1981, pp. 34-35.

"Llaguno, E. y Ceán Bermúdez, J.A., Noticias de los arquitectos..., IV, pp. 264-267; Sambricio, Carlos, «José de Hermosilla y el ideal historicista de la arquitectura de la Ilustración», Goya, 189, pp. 140-151; Rodríguez Ruiz, Delfín, «De la utopía a la Academia. El tratado de arquitectura civil de José de Hermosilla», Fragmentos, 3, 1984, pp. 57-80.

${ }_{12}$ Viaje de España, XII, Madrid 1789, edición moderna de M. Aguilar, Madrid 1947, pp. 1085-1086.

${ }^{13}$ Se halla en el Archivo General de Simancas, Gracia y Justicia, legajo 959. Fue parcialmente utilizada por Luis Sala Balust en la obra citada Visitas y reformas de los Colegios Mayores de Salamanca, pp. 82-84.
}

$A E A$, LXXVI, 2003, 302, pp. 187 a 205 
trucción de un edificio cuia admirable Arquitectura, sobre hermosear este pueblo que es tan notorio, han proporcionado subsistencia a considerable número de artesanos que, sin este recurso, hubieran sido tal vez víctima de la miseria de los años pasados». Terminaban indicando que la suspensión de la obra suponía un enorme perjuicio pues estaban prevenidos muchos materiales labrados y por labrar que se arruinarán dejándolos al descubierto de las lluvias del próximo invierno, como así lo había reconocido la Real Chancillería de Valladolid por auto del pasado mes de abril.

No convencieron estas razones transmitidas por el obispo a don Manuel de Roda, quien le escribía a 2 de julio: «Tengo por cierto que no hubo ninguna de las que alegan, sino que sólo se movieron del deseo de sovervia y pródiga ostentación. Han gastado en ella sumas muy crecidas y se han cargado de censos de mucha consideración. Para proseguirla han de gastar todavía muchísimo porque la proseguirán con la magnificencia correspondiente a lo hecho y a lo que tienen ideado». Sin embargo el obispo Beltrán era partidario de que la construcción se prosiguiese, aunque moderando sus gastos en todo lo posible, y para evaluarlos consultó a dos arquitectos locales, quienes redactaron el 21 de julio un informe sobre lo que faltaba por hacer del edificio y sobre los materiales apilados para este fin. Fueron Jerónimo García de Quiñónez, maestro entonces de las obras municipales y hombre de confianza del prelado, pues estaba terminando la acomodación del colegio de los jesuitas expulsos a seminario conciliar, y Nicolás Rodríguez Posada. Reproducimos íntegro su dictamen por considerarlo de capital importancia:

«Ilmo. Sr. Certificamos cómo habiendo pasado al Colegio maior de S. Bartolomé a la visita y reconocimiento del estado en que se alla la obra y materiales prebenidos para su entera conclusión, hallamos y reconocimos tener la maior parte de los prebenidos y dispuestos expuestos a las inclemencias de los tiempos y de querer recogerlos se seguirían maiores gastos y perjuizios. Asimismo reconocimos faltar sólo para su total y perfecta construzión en la escalera principal un pedazo de pared del lado norte, su armadura, vóbeda y asiento de mesas y pasos, el tendido de los zielos rasos de dcho. lienzo, el embaldosado del primero y segundo piso del Patio y gradas del Atrio, los tendidos y enluzidos de la Rectoral y abitaciones de quartos y comunes: como también el asiento de los balcones que están prebenidos para la librería nueva y traslado de ella, por hallarse como se halla la antigua en el sitio que ha de serbir de Cozina y Refectorio y expuesta está a un incendio por su inmediación de la provisional en una tenada que oy sirve. Por cuios motivos y razones somos de sentir serán grandes las pérdidas y más la imposibilidad de abitarse. Salamanca, 21 de julio de 1771 años».

A la vista de este informe el obispo consintió en que se continuase la obra, decisión que aprobó don Manuel de Roda en carta del día 29. Durante los cinco años siguientes se fue completando la construcción de los sectores y partes que allí se señalaron, especialmente de la escalera, pero con mucha lentitud y entre zozobras de si las reformas disciplinarias aplicadas al colegio harían posible la continuación de su existencia. En 1776 habían sido desalojados los antiguos colegiales, quedando únicamente el rector don Agustín de Emparan a la espera de que designaran la persona en la que hiciese entrega del edificio, biblioteca, alhajas y demás bienes. De un informe del visitador del colegio en 1782, don Antonio Piñuela, se desprende que el nuevo edificio se había concluido íntegramente en 1778: «Todas las oficinas se hallan aseadas, bien acondicionadas y cuidadas, existentes las alhajas de oro, plata y manillas de aljófar en los archivos del Colegio, la capilla de S. Juan de Sahún sita dentro de él... La cuenta final ya cerrada de la obra concluida del Colegio, dada por el tesorero don Josef de la Centolla, comprehensiva desde el año de sesenta en sesenta y uno hasta el de setenta y ocho en setenta y nueve monta dos cuentos setecientos cincuenta y cuatro mil y cuarenta y un reales y veintitrés maravedis de vellón» ${ }^{14}$.

${ }^{14}$ Sala Balust, Luis, Ibid., p. 442. 
Los estatutos de reforma publicados el 21 de febrero de 1777, donde se regulaba la admisión de los nuevos colegiales y becarios, permitió que el colegio se poblase de nuevo con una media de 19 colegiales por año, pero desgraciadamente esta situación duró poco pues en 1786 prácticamente el colegio se había extinguido, pasando luego el edificio al servicio de otros usos que no pretendemos relatar. Se ha solido pensar que el espléndido palacio de Anaya responde efectivamente al espíritu reformador de la Ilustración, encarnado en el reinado de Carlos III. Esta afirmación es sólo parcialmente cierta toda vez que en su construcción fue proyectada en 1760 , antes de iniciarse las reformas de los colegios mayores, correspondiendo al prurito de suntuosidad y magnificencia propias del Barroco. Así lo expresaron el rector y los colegiales de entonces, quienes desearon que el edificio que nuevamente se edificaba no sólo no desentonase de los otros monumentos de la ciudad sino que positivamente los superase. Sin embargo también es cierto que el edificio ideado por Hermosilla supuso un auténtico revulsivo frente a un barroco fundamentalmente decorativo y de oropel que había arraigado fuertemente en Salamanca de la mano de los Churriguera, de los García Quiñónes y de Simón Gavilán Tome.

José de Hermosilla, que había estado en Roma como becario de la Junta preparatoria de la Academia de San Fernando entre 1747 y 1751, conocía a la perfección la arquitectura de la Ciudad Eterna y proyectó el nuevo colegio de San Bartolomé como un palacio romano. Para ello opino que volvió la vista retrospectivamente a modelos no del barroco clasicista sino del Quinientos. El florentino Antonio da Sangallo había encontrado entonces una tipología de fachada de palacio que S. Benedetti calificó de «sintetista» o quintaesenciada, donde predominaban descarnadamente las superficies y los volúmenes frente a episodios como la articulación de los órdenes y los encuadramientos plásticos de vanos y huecos ${ }^{15}$. Es una fórmula que se encuentra en la vasta fachada del colegio salmantino. El primer cuerpo se estructura a base de cuatro ejes verticales de ventanas, entresuelos y balcones de cada lado, no articulados por pilastras sino ritmados por la equidistancia que guardan entre sí, únicamente en los extremos figuran unas pilastras aisladas de orden jónico que recorre horizontalmente toda la fachada, trabando también a ella el pórtico. Ahora bien frente a la planitud del muro de fondo destaca la plasticidad y el relieve de este pórtico, que compone el eje central y actúa como si a un palacio quinientista se le hubiera antepuesto el pórtico del Panteón de Agripa. Es tetrástilo y en sus intercolumnios diástilo, cual si Hermosilla hubiera querido proporcionarnos una lección de arquitectura vitrubiana, tanto más cuanto que a él se asciende, como si de un templo antiguo se tratase, a través de una elevada escalinata de veinte peldaños separados por un descansillo.

Las poderosas columnas del pórtico tienen el fuste liso pues «su mayor hermosura - $\mathrm{co}-$ menta Hermosilla en el Tratado de Arquitectura Civil escrito en 1750 - es la desnuda integridad de su tronco» ${ }^{16}$. Sin embargo el capitel no es el canónico del orden jónico sino el extractado del palacio de los Conservadores de Miguel Ángel, en Roma, que Hermosilla conocía muy bien por haber enviado a la Academia dibujos de su planta y alzados: es decir un capitel de volutas muy voluminosas, algo caídas hacia abajo, entre las que cuelga una guirnalda.

Hermosilla descompuso un tanto la calibrada disposición de la fachada con la adición de un ático, acaso obligado a levantar un piso superior donde instalar la nueva biblioteca del colegio. Este último piso lleva cuatro ventanas por cada lado como el primer cuerpo, pero coronadas por frontoncillos curvos, y está rematado por un macizo antepecho que ridículamente se cala y se hace balaustrada en su sector central. Los minúsculos flameros que recorren el antepecho, a todas luces desproporcionados respecto al enorme escudo de Anaya que campea en el

\footnotetext{
${ }^{15}$ Benedetti, Sandro, Fuori del Clasicismo. Sintetismo, tipologia, ragione nell'architettura del Cinquecento, Multigrafica Editrice, Roma 1984, pp. 33-61.

${ }^{16}$ Rodríguez Ruiz, Delfín, op. cit., p. 66.
}

AEA, LXXVI, 2003, 302, pp. 187 a 205 
centro, pueden ser añadidos de Sagarvinaga al proyecto de Hermosilla, si ya no es de su minerva todo este último piso.

Más sorprendente aún que la fachada es el interior del colegio. Atravesado el pórtico se accede al patio que Hermosilla concibió con atrevida originalidad como un atrio de casa romana. Efectivamente se compone de dos pisos adintelados donde resaltan absolutamente las columnas exentas, exhibidas en toda su hermosa plasticidad como genuinos soportes tectónicos. El arquitecto, asimiladas las enseñanzas habidas en Roma a mediados de siglo en que se ponían en crisis soluciones hasta entonces intocables como el pilar compuesto, había lamentado en su Tratado de Arquitectura que se hubiese perdido el uso de las basílicas primitivas «donde las naves se formaban sobre columnas» ${ }^{17}$. El primer piso es de un riguroso orden toscano, diseñado con un purismo excepcional en todos sus elementos y molduras, y el segundo de orden jónico. Lo mismo que en el pórtico los fustes son lisos, para no macular su integridad pétrea, pero en el segundo piso el capitel jónico adopta la modalidad miguelangelesca ya comentada. Por otro lado, en plena coherencia con el sistema adintelado, las cuatro crujías de este atrio, tanto las inferiores como las superiores, están cubiertas con cielos rasos, no con bovedillas de arista ${ }^{18}$.

Un patio tan radicalmente novedoso, que adelanta en décadas lo que más tarde se practicaría en el Neoclasicismo hispano, ha suscitado el interrogante sobre su posible referente inmediato. Ya don Manuel Gómez Moreno sugirió que Hermosilla había arrancado del patio del palacio de Carlos V en Granada, diseñado por Pedro Machuca, patio que había visto y dibujado cuando fue enviado por la Academia en 1766, junto con Diego de Villanueva y Pedro Arnal, a la Alhambra para levantar su planta y alzados. Si es que se acercó a Salamanca, como es de suponer, para analizar el terreno y tomar las medidas necesarias para formar el proyecto del colegio, pudo ver y examinar también el patio del colegio del Rey, de la Orden Militar de Santiago, construido al parecer por Juan Gomez de Mora, quien utilizó precozmente en él el sistema adintelado en ambos pisos, bien que empleando en ellos el mismo orden dórico sincopado ${ }^{19}$.

Del cuadrado del patio descuella por su costado occidental la enorme caja de la escalera, que ocupa buena parte de la superficie total del edificio; por eso no puede extrañar que su hipertrofiado volumen escandalizase a los que ordenaron paralizar la obra, acusándola de vana y dispendiosa ostentación. No es precisamente esta escalera - lo último que se construyó- la parte más acertada del colegio, todo lo contrario disuena su estilo falsamente clásico de la novedad radical con que Hermosilla se desligó de la corriente barroca clasicista. La escalera arranca de un mezquino triple hueco horadado en la crujía occidental del atrio y ramplonamente moldurado, triple hueco que se corresponde con las rampas de subida, una en el centro hasta alcanzar la meseta y otras dos que desde allí se bifurcan. Las paredes de la caja se estructuran a media altura mediante una secuencia de arcos ciegos, tres por cada lienzo de muro, que alberga los marcos rectangulares de las ventanas, arcos separados por un orden corintio con su correspondiente entablamento. Sobre éste se levanta una fila de lucernarios de los que surge una bóveda esquifada hecha de ladrillo tabicado y enrasado. Es posible, aunque no existe documentación para probarlo, que toda la escalera, al ser lo último que se completó tras la interrupción de la obra, hubiera sido no sólo realizada sino ideada por Juan de Sagarvinaga, desentendido ya Hermosilla del edificio, pues su estilo se corresponde con lo obrado en otros sitios por el maestro de obras de la catedral de Salamanca.

Alfonso Rodríguez G. De Ceballos

${ }^{17}$ Ibid., p. 72

${ }^{18}$ Catálogo monumental de España. Provincia de Salamanca, Ministerio de Educación y Ciencia, Madrid 1967, p. 305.

${ }^{19}$ Este patio subsistió hasta comienzos del siglo xx y aún existen fotografías de él.

AEA, LXXVI, 2003, 302, pp. 187 a 205 